whether a shorter course of radiation therapy could also provide effective pain and narcotic relief. Patients with breast or prostate cancer who had one to three sites of painful bone metastases were randomly assigned to one of two treatment regimens. A total of 455 patients received $8 \mathrm{~Gy}$ of radiation therapy in a single dose (8-Gy arm) and 443 patients received $3 \mathrm{~Gy}$ in 10 doses over 2 weeks, up to a total of 30 Gy (30-Gy arm). At 3 months' follow-up, no differences in pain relief, narcotic relief or incidence of pathologic fractures were reported between patients in the 8-Gy and 30-Gy arms.

Twice as many patients in the 8-Gy arm required retreatment compared with the 30-Gy arm, but the authors note that this problem could be counterbalanced by the lower levels of acute toxicity observed in patients receiving a single dose of radiation therapy.

Original article Hartsell WF et al. (2005) Randomized trial of short- versus long-course radiotherapy for palliation of painful bone metastases. J Natl Cancer Inst 97: 798-804

\section{Importance of testosterone dosage for bone mineral density}

Bone mineral density (BMD) in androgendeficient (AD) men was the focus of a recent retrospective study in Australia, in which researchers sought to assess the impact of the quality of testosterone replacement given to patients on their BMD.

Aminorroaya et al. analyzed BMD data from 169 men with organic androgen deficiency (excluding men with age-related "hypogonadism"), who were receiving different dose regimens of androgen-replacement therapy. Patients were initially categorized as untreated, partially treated, or well treated. The men underwent cross-sectional dual-energy X-ray absorptiometry scanning in order to measure their BMD. Following this, they were optimally treated with $800 \mathrm{mg}$ testosterone implants, and their BMD was measured again at 2-3 year intervals.

The authors found that the initial ageadjusted BMD of well-treated $A D$ men was significantly better than that of men who had been partially treated or untreated, and their BMD was found to be comparable to that of healthy non-AD men. Longitudinal analysis of dual-energy X-ray absorptiometry scans carried out 2 years following the initial evaluation revealed that men who were untreated at the time of the first scan demonstrated the greatest gains in BMD over time. They also achieved a similar BMD to that of age-matched nonAD men.

In conclusion, the authors state that a consistently administered, optimal dose of testosterone-replacement therapy is able to normalize the BMD of AD men, independently of the type or etiology of their androgen deficiency.

Original article Aminorroaya A et al. (2005) Adequacy of androgen replacement influences bone density response to testosterone in androgen-deficient men. Eur J Endocrinol 152: $881-886$

\section{Effects of relaxin on elastin metabolism in pelvic tissue of women with stress urinary incontinence}

Chen et al. hypothesize that a common process leading to stress urinary incontinence (SUI) in women is the degradation of both collagen and elastin in the extracellular matrix of pelvic tissues, both of which provide the pelvic floor with the ability to recoil after physical stress and distension. The authors investigated the effects of relaxin-a peptide hormone produced by the corpus luteum, known to be important in cervical ripening-on elastin proteases and their corresponding inhibitors, in cultured vaginalwall fibroblasts.

This small preliminary study enrolled 10 women, four with SUI and six asymptomatic control subjects. Equal numbers of women in each group were in the proliferative and secretory phases, respectively, of their menstrual cycles. Fibroblasts were obtained and the effects of different concentrations of relaxin on expression of elastolytic matrix metalloproteinases (MMP) 2 and 9, tissue inhibitors of metalloproteinases (TIMP) 1 and 2, human neurophil elastase and $\alpha-1$ antitrypsin were studied in vitro.

As relaxin concentrations increased, expression of MMPs increased in all fibroblast cultures except those from women 